This is the final peer-reviewed accepted manuscript of:

TCAD investigation on hot-electron injection in new-generation technologies

S.Reggiani M.Rossetti A.Gnudi A.N.Tallarico A.Molfese S.Manzini R.Depetro G.Croce E.Sangiorgi C.Fiegna

“Microelectronics reliability", Volumes 88-90, September 2018, Pages 1090-1093

The final published version is available online at:

https://www.sciencedirect.com/science/article/pii/S0026271418306590 (C) [2018].

This manuscript version is made available under the Creative Commons AttributionNonCommercial-NoDerivs (CC BY-NC-ND) License 4.0 International 


\title{
TCAD investigation on hot-electron injection in new-generation technologies
}

\author{
S. Reggiani ${ }^{\mathrm{a}}$, M. Rossetti ${ }^{\mathrm{c}}$, A. Gnudi ${ }^{\mathrm{a}}$, A. N. Tallarico ${ }^{\mathrm{b}}$, A. Molfese ${ }^{\mathrm{c}}$, \\ S. Manzinic ${ }^{c}$, R. Depetro ${ }^{\text {c }}$, G. Croce ${ }^{\text {c }}$, E. Sangiorgi ${ }^{b}$, C. Fiegna $^{\mathrm{b}}$ \\ ${ }^{a}$ ARCES and DEI, University of Bologna, Bologna, Italy \\ ${ }^{\mathrm{b}}$ ARCES and DEI, University of Bologna, Cesena, Italy \\ c Technology R\&D, STMicroelectronics, Agrate Brianza, Italy
}

\begin{abstract}
The hot electron injection model presently available in the TCAD tools has been investigated against experiments on new test devices to the purpose of gaining an insight on its predictability in the context of the newgeneration technologies. The study has been carried out on electron emission in extremely high electric fields, as expected in power LDMOS devices at the onset of avalanche breakdown, reaching for the first time injection probabilities as high as 0.01 . The numerical analysis clearly showed that the new $\mathrm{Si} / \mathrm{SiO}_{2}$ interfaces experience different features with respect to the old ones. The TCAD method based on the deterministic solution of the Boltzmann equation can accurately capture such effects.
\end{abstract}

\section{Introduction}

One of the key challenges in building devices for analog and high-voltage applications in advanced technology platforms is given by their reliability concerns. The scaling of CMOS devices inevitably leads to hot-carrier-stress instability and charge injection [1]. This leads to the need of predictive models suitable for the design stage.

Recently, a TCAD approach has been proposed based on the deterministic solution of the Boltzmann equation through the Spherical Harmonic Expansion (SHE-BTE) [2]. The model was implemented in the Synopsys TCAD tool [3] and was shown to fairly reproduce the charge-injection experiments reported by Ning [4]. It accounts for the microscopic scattering mechanisms caused by acoustic and intervalley phonons, ionized impurities, and impact ionization and uses the band-structure quantities obtained from the nonlocal empirical pseudopotential method for relaxed silicon up to the fourth band, which starts at about 3.23 $\mathrm{eV}$. It is expected that more bands appear at higher energies, but the SHE-BTE solution is assumed to be accurate for the description of the relevant injection probabilities as they are mostly characterized by the distribution function close to the $\mathrm{Si} / \mathrm{SiO}_{2}$ energy barrier $(3.1 \mathrm{eV})$ further corrected by the barrier-lowering effects when high fields are applied $[4,5]$. The tail of the distribution function usually shows an exponential decrease, thus the description of the bands and scattering rates at energies higher than about $4 \mathrm{eV}$ should not significantly affect the hot-carrier injection predictions. In addition, the SHE solution implemented in [3] is based on the lowest order expansion, which was recently compared to the high-order SHE results showing that reasonable electron energy distribution is expected for high-field conditions [6].

The injection process was modeled following [5], with a compact equation used to calculate the gate current density accounting for the image force, the scattering in the oxide conduction band, the transmission probability of electrons by tunneling or emitted over the barrier. The injection model based on the BTE-SHE solution was recently applied to nanoscale devices to verify its predictions on inhomogeneous cases, and it demonstrates to reproduce the Monte Carlo gate current densities and the 

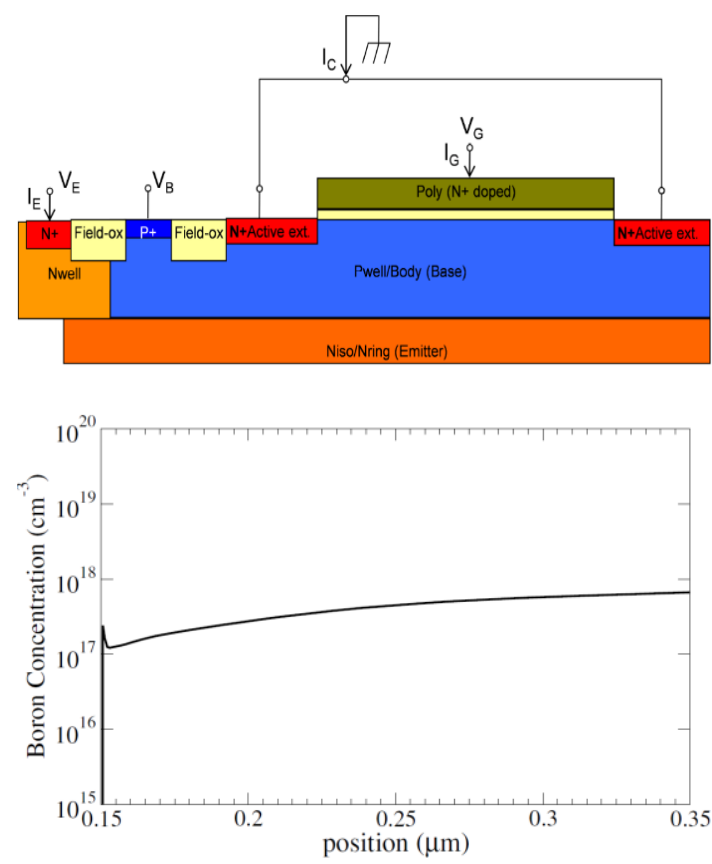

Fig. 1. Top: Schematic view and bias conditions of the nchannel MOSFET with sub-surface emitter region used to inject carriers in the MOSFET substrate. Bottom: Simulated Boron doping profile in the substrate (gate oxide interface at $0.15 \mu \mathrm{m}$ ).

experimental gate-to-drain current ratios without any calibration [7]. Thus, it is expected to give a rigorous prediction of hot-carrier injection probability even in analog and power devices.

In this work, the Synopsys-TCAD hot-electron injection model has been adopted to reproduce the experiments of new test devices. The numerical analysis clearly showed that the new $\mathrm{Si} / \mathrm{SiO}_{2}$ interfaces experience different features with respect to the old ones.

\section{Test structures and experiments}

A conventional n-channel MOSFET fabricated with a BCD process has been used with an additional sub-surface emitter region to control the electron injection from the forward-biased emitter-base junction into the MOSFET substrate (Fig. 1). The device has a $7 \mathrm{~nm}$-thick thermally grown gate oxide, annealed in nitrogen monoxide. Thus, the interfacial nitrogen concentration is expected to generate a $\mathrm{SiON} / \mathrm{Si}$ interface. The body (p-well) of the MOSFET has a near-surface boron concentration of about $3 \times 10^{17}$

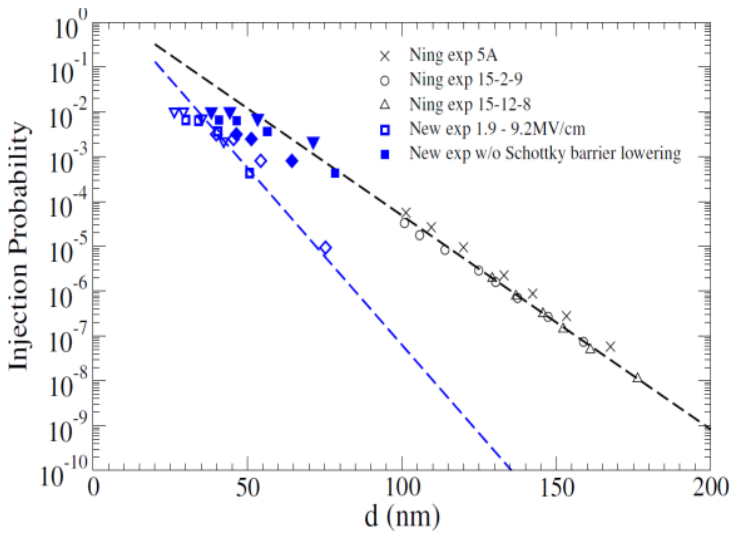

Fig. 2. Injection probability as a function of the distance $d$ from the $\mathrm{Si} / \mathrm{SiO}_{2}$ interface. Black symbols: Ning experiments [4]. Open blue symbols: experiments carried out on the new devices for different $E_{o x}$, reported as functions of $d$ accounting for barrier lowering. Closed blue symbols: new experiments as functions of $d$ without barrier lowering. Dashed lines where calculated as $P_{i n j}=$

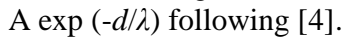

$\mathrm{cm}^{-3}$ (Fig. 1, bottom).

The experiments are performed at constant temperature $\mathrm{T}=25{ }^{\circ} \mathrm{C}$, controlled by a thermo-chuck. The device has been biased at zero drain-to-source voltage, above threshold $\left(V_{G}=1.4 \div 7 \mathrm{~V}\right)$ to modulate the oxide electric field $\mathrm{E}_{\mathrm{ox}}$, and with different negative body voltage $\left(V_{B}=0 \div-5 \mathrm{~V}\right)$ to modulate the depletion region below the gate. Electrons have been injected by forcing $I_{E}=-100 \mu \mathrm{A}$, the body voltage is swept from 0 to $-5 \mathrm{~V}$ and the collector, body and gate currents are monitored. The injection probability $\left(P_{i n j}\right)$ is equal to the ratio between the gate and collector current.

For a preliminary analysis, $P_{i n j}$ measured for three different $V_{G}$ have been reported in Fig. 2 with blue squares, triangles and diamonds, respectively, as function of the distance $d$ from the $\mathrm{Si} / \mathrm{SiO}_{2}$ interface where the potential energy in the substrate is equal to the barrier height. The drift-diffusion TCAD simulations, carried out by solving the electron and hole transport equations accounting for the corresponding impact-ionization generation terms, have been calibrated against the $I_{C}-V_{B}$ experiments, as they clearly show the effect of avalanche with a significant increase of $I_{C}$ with decreasing $V_{B}$ leading to $I_{C}$ even larger than $I_{E}$ for $V_{B}<-4.5 \mathrm{~V}$. The calibrated TCAD simulations have been used to extract $d$ at different $V_{B}$. Due to the larger doping concentration, a significant reduction of $d$ is experienced by the new 
devices. The barrier height as a function of $E_{o x}$ has been initially modelled according to [4] because it showed good predictions on devices with different doping profiles. By modelling the emission probability with the expression $P_{i n j}=\mathrm{A} \exp (-d / \lambda)$, with A and $\lambda$ fitting parameters (dashed curves in Fig. 2), the new data (open blue symbols) clearly show a different barrier dependence with respect to Ning experiments (open black symbols). It is worth noting that the $\lambda$ parameter in [4] is the mean free path for opticalphonon scattering in the depleted silicon region (those electrons lucky enough to escape collision in silicon are emitted in the oxide). No relevant differences are expected for the new data with respect to the old ones, as it should not depend on the doping concentration in the silicon substrate. If this is the case, the difference should be ascribed to the determination of $d$, which is a function of the barrier height. The latter can be ascribed to the nitrided gate oxide adopted in the new devices $[8,9]$. By assuming a zero Schottky-barrier lowering, a larger $d$ could be obtained for the new data in accordance with Ning results (Fig. 2, closed blue symbols).

\section{TCAD simulations of the SHE-BTE}

The accurate simulation of hot electron injection is a complex task as underlined in [5]. At high energies, the optical-phonon and impact-ionization scattering are expected to determine the shape of the distribution tail, thus we checked the parameters in the TCAD model and changed the impact-ionization rate consistently with the data reported in $[5,10]$ and references therein (Fig. 3). As the same band structure and phonon rate of [5] have been used, the impact-ionization scattering rate has been defined consistently: the reported manythreshold model is able to fit the impact-ionization coefficient, the impact-ionization quantum yield, and the data from soft X-ray photo-emission spectroscopy. A few sets of data reported in [10] are in fair agreement with experiments, which are reported in Fig. 3 as reference. The SHE-BTE scattering rates nicely compare with the Monte Carlo data up to $5 \mathrm{eV}$.

To this purpose, the expression provided by [10] has been used for the ionization rate $1 / \tau_{\mathrm{ii}}(E)$ as a function of electron energy $E$ :

$$
\frac{1}{\tau_{i i}}=\sum_{i=1}^{3} \theta\left(E-E_{t h}^{(i)}\right) P^{(i)}\left(\frac{E-E_{t h}^{(i)}}{1 \mathrm{eV}}\right)^{2}
$$
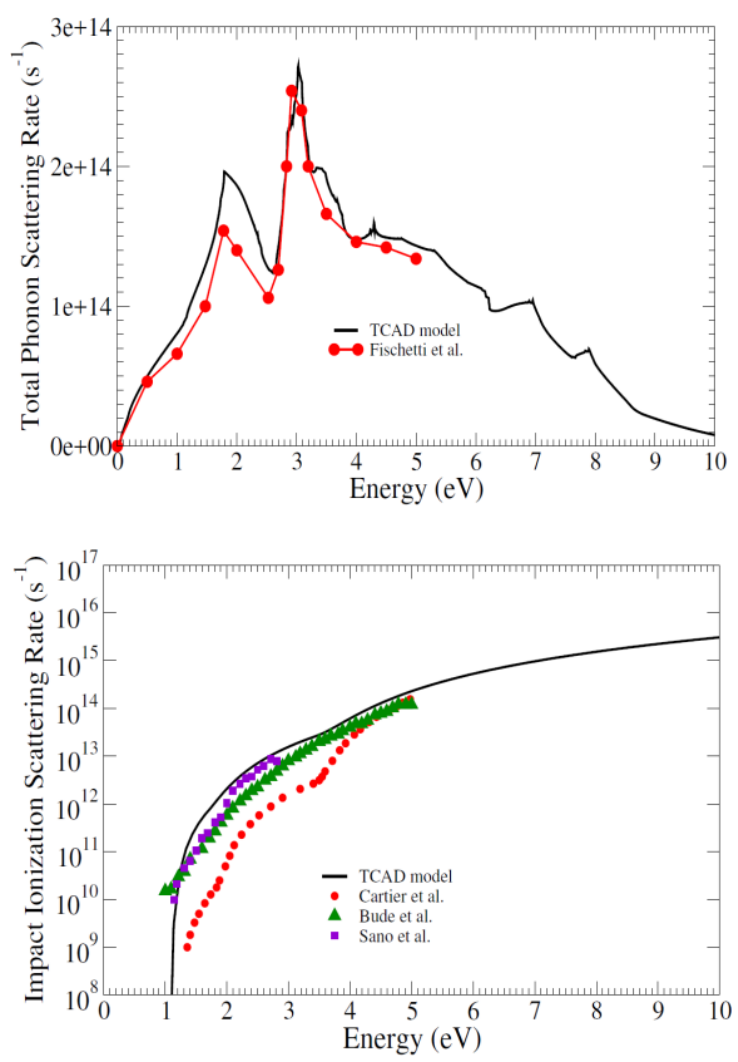

Fig. 3. (Top) Total electron-phonon and (bottom) impactionization scattering rates computed with SHE-BTE in comparison with the ones adopted in [5] and [10]. Default parameters are used for phonon rate, while a different parameter set is used for the second one.

where, for $i=1,2$ and $3, E_{\text {th }}^{(i)}=1.12,1.75$ and $3.45 \mathrm{eV}$, $P^{(i)}=8 \times 10^{11}, 6 \times 10^{12}$ and $5 \times 10^{13} \mathrm{~s}^{-1}$, and $\theta$ is the step function.

The structure of the device reported in Fig. 1 has been used to create the simulation deck for the new test structures and the devices reported by Ning ("15-12-8" in [4]). In Fig. 4, the electron energy distributions at the $\mathrm{Si} / \mathrm{SiO}_{2}$ interface obtained by the SHE-BTE solution are compared for both devices at the maximum $V_{G}$ and $V_{B}$. The tail of the distribution function in the new structure is significantly higher than the old device due to the larger doping concentration. As expected, the new devices cover a range of hot-carrier energies which was not reached by the old structures.

\section{TCAD calculations of the gate current}




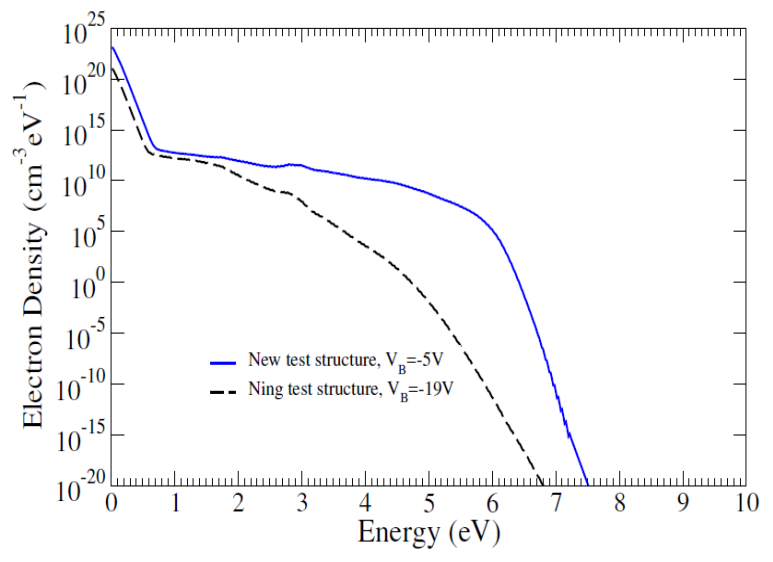

Fig. 4. Electron density as a function of energy computed with the SHE-BTE code in the new and old test structures at the maximum body biases.

The implemented gate current model available in [3] covers tunnelling and thermionic emission components and accurately fits the Ning experiments as shown in [2]. It takes into account the image potential barrier lowering and the scattering probability within the oxide. More specifically, the probability of electrons moving from silicon to oxide without scattering is given by $P_{i n s}=\exp \left(-r_{0} / \lambda_{i n s}\right)$, with $r_{0}$ the distance of the barrier peak in the oxide, and $\lambda_{i n s}$ the mean free path in the insulator. The gate current is calculated from the solution of the SHE-BTE and is proportional to $P_{i n s}$. By observing the results of Fig. 2, the comparison between new and old experiments provides the indication that a much smaller barrier lowering is experienced in the new structures, thus the corresponding image-potential barrier-lowering model $[2,5]$ has been switched off in the TCAD simulations. A slight calibration of the barrier height has been applied $\left(E_{B O}=3.0\right.$ instead of $\left.3.1 \mathrm{eV}\right)$. In addition, a significant oxide scattering has been assumed, leading to a strongly reduced mean free path. In Fig. 5, the TCAD results of $P_{i n j}$ as a function of $E_{o x}$ by ignoring the barrier-lowering effect on the barrier height (but keeping the oxide scattering effect) nicely reproduce the measured data for all biases, especially at the higher injection conditions, while numerical data assuming the barrier lowering show a clear overestimation at larger biases. The best fitting is obtained by using $\lambda_{\text {ins }}=0.3$ instead of $2 \mathrm{~nm}$. In Fig. 6, the TCAD simulations on the old device by ignoring the barrier-lowering effect

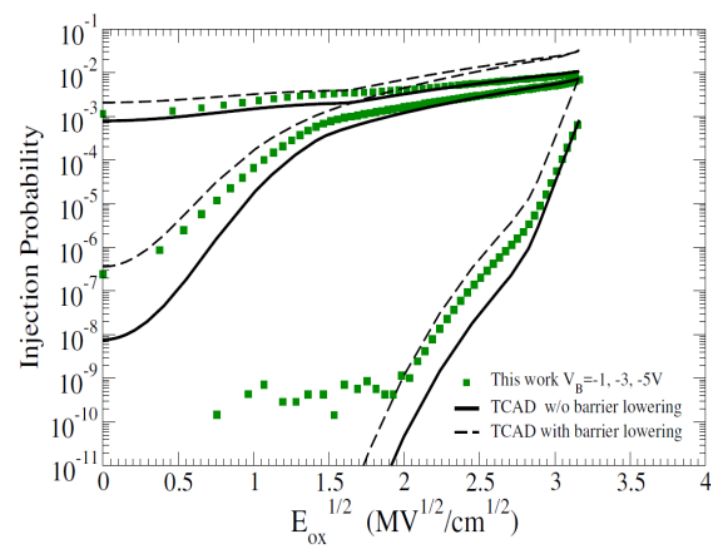

Fig. 5. Measured and simulated probability of injection into $\mathrm{SiO}_{2}$ as a function of the square root of the oxide field at three different body biases. Measured data (symbols) show a weak dependence on $E_{o x}$ at high injection conditions, which is in accordance with the results shown in Fig. 2. Solid lines: TCAD predictions without barrier lowering. Dashed lines: TCAD predictions with barrier lowering. TCAD results with barrier lowering switched off show the best fitting with experiments.

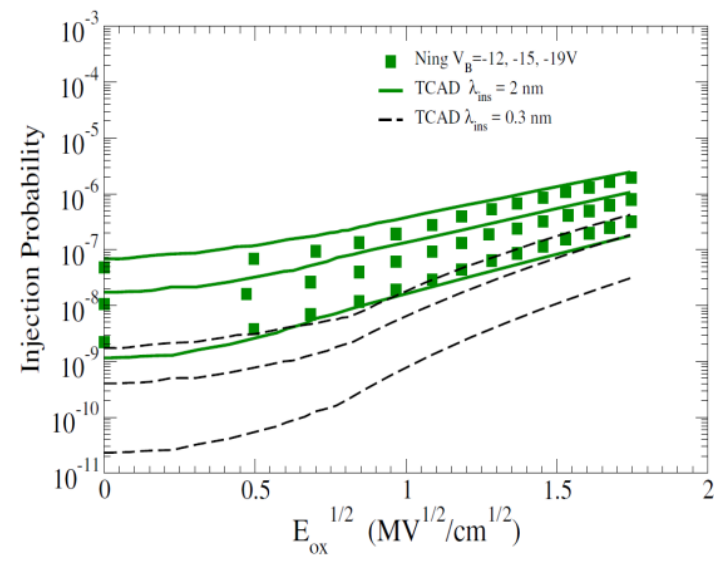

Fig. 6. Measured and simulated probability of injection into $\mathrm{SiO}_{2}$ as a function of the square root of the oxide field at three different body biases. Symbols: "15-12-8" Ning experiments [5], showing an exponential increase with the square root of $E_{o x}$. Solid lines: TCAD results with barrier lowering switched off and the default value for $\lambda_{\text {ins. }}$. Dashed lines: TCAD results with barrier lowering switched off and $\lambda_{i n s}=0.3 \mathrm{~nm}$. The best fitting with experiments is obtained by using the default value for the insulator mean free path. 
on the barrier height have been reported as functions of $E_{o x}$ : the dependence on $E_{o x}$ is still high enough to accurately fit Ning experiments, provided that the usual value of $\lambda_{\text {ins }}$ is adopted $[2,3]$. The obtained results showed that, on the one hand, the barrier lowering effect is not observed at very high $E_{o x}$ and is not necessary to fit the experiments. On the other hand, the scattering rate at the oxide barrier seems to be much more relevant in the new devices with respect to the old ones as different values of the mean free path have been extracted against experiments, which might be related to the different process features such as oxide growth conditions and chemical composition.

\section{Conclusions}

In this work, the hot electron injection model presently available in the TCAD tools has been investigated against experiments on new test devices to the purpose of gaining an insight on its predictability in the context of the new-generation BCD technologies. The study has been carried out on electron emission in extremely high electric fields, as expected in power LDMOS devices at the onset of avalanche breakdown, reaching for the first time injection probabilities as high as 0.01 . The numerical analysis clearly showed that the new $\mathrm{Si} / \mathrm{SiO}_{2}$ interfaces experience different features with respect to the old ones. The TCAD method based on the deterministic solution of the Boltzmann equation can accurately capture such effects, confirming to be an interesting approach for future analyses.

\section{Acknowledgments}

This work was partially supported by ECSEL 2014-2653933: R2POWER300 "Preparing R2 extension to 300mm for BCD Smart Power and Power Discrete" and by H2020-EU ECSEL 737417: R3-PowerUP "300mm Pilot Line for Smart Power and Power Discrete".

\section{References}

[1] L. Atzeni and M. Rossetti, "Effect of Reverse Body Bias on Hot Carrier Induced Degradation of n-MOSFETs", IEEE Trans. DMR 18, p. 80, 2018.

[2] S. Jin, A. Wettstein, W. Choi, F. M. Bufler and E. Lyumkis, "Gate Current Calculations Using Spherical Harmonics Expansion of Boltzmann Equation", IEEE SISPAD 2009, p. 202.

[3] Synopsys Inc., Sentaurus Device User Guide M-2016.12,
2016.

[4] T.H. Ning, C.M. Osburn and H. N. Yu, "Emission probability of hot electrons from silicon into silicon dioxide", J. Appl. Phys. 48, p. 286, 1997.

[5] M. V. Fischetti, S. E. Laux and E. Crabbé, "Understanding hot-electron transport in silicon devices: Is there a shortcut?", J. Appl. Phys. 78, p. 1058, 1995.

[6] S. Jin, S.-M. Hong and C. Jungemann, "An Efficient Approach to Include Full-Band Effects in Deterministic Boltzmann Equation Solver Based on High-Order Spherical Harmonics Expansion", IEEE Trans. on El. Dev. 58, p. 1287, 2011.

[7] A. Zaka, Q. Rafhay, M. Iellina, P. Palestri, R. Clerc, D. Rideau, D. Garetto, E. Dornel, J. Singer, G. Pananakakis, C. Tavernier and H. Jaouen, "On the accuracy of current TCAD hot carrier injection models in nanoscale devices", Solid-State Electronics 54, p. 1669, 2010.

[8] B. Kaczer, V. Arkhipov, M. Jurczak, and G. Groeseneken, "Negative Bias Temperature In-stability (NBTI) in $\mathrm{SiO} 2$ and $\mathrm{SiON}$ gate dielectrics understood through disorder-controlled kinet-ics", Microelectronic Engineering 80, p. 122, 2005.

[9] C. Y. Ng, T. P. Chen and C. H. Ang, "Dependence of barrier height and effective mass on nitrogen concentration at $\mathrm{SiOxNy} / \mathrm{Si}$ interface and gate oxide thickness", Smart Mater. Struct. 15, p. S39, 2006.

[10] E. Cartier, M. V. Fischetti, A. Eklund, and F. R. McFeely, "Impact ionization in silicon", Appl. Phys. Lett. 62, 3339-3341, 1993. 